\title{
Compactness in the choice and game theories: a characterization of rationality
}

\author{
Athanasios Andrikopoulos
}

Received: 3 March 2013 / Accepted: 12 March 2013 / Published online: 27 March 2013

(C) SAET 2013

\begin{abstract}
Compactness is an important topological property as it enables us to apply minimax theorems in economic theory. The theory of optimal choice sets is a solution theory that has a long and well-established tradition in social choice and game theories. A general solution concept of choice problems when the set of best alternatives does not exist (this problem occurs when the preferences yielded by an economic process are cyclic) is the Schwartz set. This set is one of the most popular solution concepts since it insures rationality. The Schwartz set is equivalent to the admissible set that appears in the game theory literature. The present note shows that the feasible set is compact if and only if every generalized upper tc-semicontinuous preference has non-empty Schwartz (admissible) set.
\end{abstract}

Keywords Schwartz set $\cdot$ Admissible set $\cdot$ Maximal element $\cdot$ Minimal undominated set

JEL Classification $\quad \mathrm{C} 02 \cdot \mathrm{C} 60$

\section{Introduction}

The concept of compactness is an extension of the benefits of finiteness ${ }^{1}$ to infinite sets. ${ }^{2}$ Most properties of compact sets in economic theory are analogous to the

\footnotetext{
1 Any topology on a finite set is compact, but a finite set is usually considered to be a discrete topological space. Here is why: (i) if a set $D$ has a discrete topology, then $D$ is compact if and only if $D$ is finite; (ii) a topology on a finite set $D$ is Hausdorff if and only if it is the discrete topology; (iii) any function from a space with the discrete topology is continuous.

${ }^{2}$ We often call an infinite set itself a compact set when it is not important what topology is being used.
}

A. Andrikopoulos $(\varangle)$

Department of Economics, University of Ioannina, Ioannina, Greece

e-mail: aandriko@cc.uoi.gr 
properties of finite sets which are quite trivial. For example, the existence theorems of maximal elements are useful and important tools to prove the existence of non-empty choice sets or equilibrium existence theorems of mathematical economies and generalized games in different underline spaces. ${ }^{3}$ Therefore, we are often interested in conditions under which we can be sure that a preference achieves a maximal element on the feasible set. ${ }^{4}$ If the feasible set is finite, a sufficient condition for the existence for such a maximal element is the preference to be acyclic. However, the same statement remains true if finiteness is replaced by compactness and the preference is upper semicontinuous. 5

A classical result of Sloss (1971), Brown (1973) and others states that any upper semicontinuous acyclic binary relation defined on a compact set has a maximal element. A converse result of Sloss and Brown is that of Gutiérrez (2009, Theorem 2.1), which says that: if the compactness from the feasible set is violated, then there exists a preference (here preference means asymmetric and negative transitive binary relation) such that for every feasible element there is one that is better according to this preference. That is, the feasible set has no maximal element with respect to this preference. Since asymmetry and negative transitivity imply acyclicity, the aforementioned results of Sloss, Brown and Gutiérrez, provide a characterization of compactness as follows: the feasible set is compact if and only if there is a maximal element for any upper semicontinuous acyclic binary relation. This characterization requires the binary relation to be acyclic. However, in collective choice problems (ranking of social preferences, voting in committees, etc.), cyclicity is a plausible assumption as, for example, the Condorcet Paradox shows. For this reason, a more general framework is needed to characterize compactness.

The classical rationality conditions in choice theory formalize the thesis that to choose rationally is to choose in such a way that no other choice would have been better, or preferable. That is, each individual makes choices by selecting, from each feasible set of alternatives, those which maximize his own preference relation. On the other hand, one of the most common assumptions made in game theory is that rationality implies that every player maximizes his own payoff. In a stricter sense, it implies that every player always maximizes his utility, thus being able to perfectly calculate the probabilistic result of every action. In any case, the choice set, from a given potential set, is the set of maximal elements under a dominance relation. But, the set of maximal elements is often empty. In this case, it is important to specify criteria that will provide reasonable sets of alternatives as solutions. In the choice and game theories, a number of theories, called general solution theories, have been proposed to take over the role of maximality in the absence of maximal elements. One of the most important general solution concepts is the Schwartz set. An equivalent notion to the Schwartz set in game theory is that of the admissible set (see

\footnotetext{
3 See for instance, Araujo et al. (2004), de Castro et al. (2011), Sánchez et al. (2003), Prokopovych (2011) and Yannelis and Prabhakar (1983).

4 The feasible set is the set of all alternatives (commodity bundles or payoff outcomes) that are possible solutions.

5 Since a finite set is considered as a discrete topological space, the continuity assumptions posed for the infinite case also hold in the finite case.
} 
Kalai and Schmeidler 1977). The admissible set concept can be applied to a host of game-theoretic situations, ranging from non-cooperative games, where a coalition consists of an individual player, to fully cooperative games, where any coalition can be allowed.

On the other hand, to face the problem of characterization of the existence of non-empty choice sets, the standard approach is to assume upper semicontinuous preferences. A number of more general concepts of continuity have been introduced in the literature in connection with the problem of the existence of maximal elements. Alcantud (2002) defines the notion of upper tc-semicontinuity which is extended by Andrikopoulos (2007) to the notion of generalized upper tc-semicontinuity.

In this study, we characterize the compactness of the feasible set in a general framework, where "preference" means arbitrary binary relation. We prove that the feasible set is compact if and only if every generalized upper tc-semicontinuous preference has non-empty Schwartz (admissible) set.

\section{Notation and definitions}

Let $X$ be a (finite or infinite) non-empty set of alternatives, and let $R \subseteq X \times X$ be a preference on $X$. We sometimes abbreviate $(x, y) \in R$ as $x R y$. The asymmetric part $P(R)$ of $R$ is given by: $(x, y) \in P(R)$ if and only if $(x, y) \in R$ and $(y, x) \notin R$. The complement of $R$ is denoted by $R^{\mathrm{c}}$ : For all $x, y \in X, R^{\mathrm{c}}=\{(x, y) \mid(x, y) \notin R\}$. The relation $R$ is asymmetric if and only if $x R y$ implies $(y, x) \notin R$. We say that $R$ is transitive if for all $x, y, z \in X,(x, z) \in R$ and $(z, y) \in R$ imply that $(x, y) \in R$. If in the definition of a transitive preference we replace $R$ with $R^{\mathrm{c}}$, we get the notion of negative transitive preference. The transitive closure of $R$ is denoted by $\bar{R}$ : For all $x, y \in X,(x, y) \in \bar{R}$, if there exist $K \in N$ and $x_{0}, \ldots, x_{K} \in X$ such that $x=x_{0}$, $\left(x_{k-1}, x_{k}\right) \in R$ for all $k \in\{1, \ldots, K\}$ and $x_{K}=y$. A subset $Y \subseteq X$ is an $R$-cycle if, for all $x, y \in Y$, we have $(x, y) \in \bar{R}$ and $(y, x) \in \bar{R}$. We say that $R$ is acyclic if there does not exist an $R$-cycle. $\mathcal{M}(X, R)$ denotes the elements of $X$ that are $R$-maximal in $X$, i.e., $\mathcal{M}(X, R)=\{x \in X \mid$ for all $y \in X, y R x$ implies $x R y\}$. A subset $A \subseteq X$ is $R$-undominated if and only if for no $x \in A$ there is a $y \in X \backslash A$ such that $y R x$. An $R$-undominated set is minimal if none of its proper subsets has this property.

Let $R$ be a preference defined on a topological space $(X, \tau)$. The preference $R$ is upper semicontinuous (resp. upper tc-semicontinuous) if for each $x \in X$ the set $\{y \in X \mid x R y\}$ (resp. $\{y \in X \mid x \bar{R} y\}$ ) is open. $R$ is generalized upper tc-semicontinuous if for each $x \in X$ the set $\{y \in X \mid x P(\bar{R}) y\}$ is open. The space $(X, \tau)$ is compact if for each collection of open sets which cover $X$ there exists a finite subcollection that also covers $X$.

\section{The main result}

The usual definition of compact spaces is based on open sets and unions. This definition, by applying de Morgan's laws, can be written using closed sets and intersections. Recall that a nest is a family of sets which is linearly ordered by inclusion. 
Nest characterization of compactness (Kelley 1955, Page 163). A topological space $(X, \tau)$ is compact if and only if each nest of closed non-empty sets has non-empty intersection.

A choice function is a functional relationship, $\mathcal{C}: \mathcal{P}(X) \rightarrow \mathcal{P}(X)$ such that, for every $A \in \mathcal{P}(X), \mathcal{C}(A)$ is a non-empty subset of $A$, which represents those alternatives chosen by the individual or society. The traditional choice-theoretic approach takes behavior as rational if there is a binary relation $R$ such that for all non-empty subsets of $X, \mathcal{C}(A)=\mathcal{M}(A, R)$. To deal with the case where the set of maximal elements is empty, Schwartz has proposed the following general solution concept:

Generalized Optimal-Choice Axiom $(\mathcal{G O C H \mathcal { A }})$ (Schwartz 1986, Page 142). For each $A \subseteq X, \mathcal{C}(A)$ is equivalent to the union of all minimal $R$-undominated subsets of $A$.

The Schwartz set is the choice set from a given set specified by the $\mathcal{G O C H} \mathcal{A}$ condition. An equivalent notion of Schwartz set in game theory, called admissible set, was introduced independently by Kalai and Schmeidler (1977) as follows: Given a set of alternatives $X$ and a binary relation $R$ on $X$, the admissible set of the pair $(X, R)$ is defined to be the set of maximal elements with respect to the transitive closure of $R{ }^{6}$ The authors show that existing solutions in game theory and mathematical economics are special cases of the admissible set. More specifically, the admissible set coincides with the core of an $n$-person cooperative game without side payments with the Nash equilibria of a game in the normal form, and it contains the competitive equilibrium prices in the case of an exchange economy with finitely many commodities and traders.

To simplify the notation, in what follows, $S(X, R)$ denotes the Schwartz set with respect to a preference relation $R$ defined over a set of alternatives $X$.

Theorem The feasible set is compact if and only if any generalized upper tcsemicontinuous preference has non-empty Schwartz (admissible) set.

Proof To prove sufficiency, suppose that $X$ is a non-compact set. Then, we must prove that there exists a generalized upper tc-semicontinuous preference $R$ in $X$ such that $S(X, R)=\emptyset$. Since $X$ is non-compact, according to the nest characterization of compactness, there exists a well-ordered decreasing family $\left\{F_{i} \mid i \in I\right\}$ of non-empty closed subsets of $X$ such that its intersection $\bigcap_{i \in I} F_{i}=\emptyset$.

Let $R^{*}$ be the binary relation which is defined in Gutiérrez (2009, Page 132), that is:

$y R^{*} x$ if there is $k \in I$ such that $y \in F_{k}$ and $x \notin F_{k}$.

We will show that $R^{*}$ is a generalized upper tc-semicontinuous preference such that $S\left(X, R^{*}\right)=\varnothing$, and proving this will complete the sufficiency part. Clearly, $R^{*}$ is asymmetric and negative transitive. It follows that $R^{*}$ is acyclic. Since, for each $x \in X$ the set $\left\{y \in X \mid x R^{*} y\right\}$ is open, $P\left(\overline{R^{*}}\right)=\overline{R^{*}}$ and for each $t \in\left\{y \in X \mid x \overline{R^{*}} y\right\}$ the inclusion $\left\{y \in X \mid t R^{*} y\right\} \subseteq\left\{y \in X \mid x \overline{R^{*}} y\right\}$ always holds, we conclude that $R^{*}$ is generalized upper tc-semicontinuous. Suppose that $S\left(X, R^{*}\right) \neq \emptyset$. We will show that this leads to a contradiction. Indeed, let $x \in S\left(X, R^{*}\right)$. Then, there exists a minimal $R$ undominated subset $D$ of $X$ such that $x \in D$. There are two cases to consider depending

6 The equivalence of the Schwartz and admissible sets emerges from Andrikopoulos (2012, Theorem 19). 
on whether $D=\{x\}$ or not. In the case where $D=\{x\}$, we have $(y, x) \notin R^{*}$ for all $y \in X$. Choose a $k \in I$ and a $y \in F_{k}$. It follows by $(y, x) \notin R^{*}$ that for each $i \in I$, either $y \notin F_{i}$ or $x \in F_{i}$. Hence, since $y \notin F_{k}$ is false, we conclude that $x \in F_{k}$. Therefore, $x \in F_{i}$ for all $i \in I$ which contradicts $\bigcap_{i \in I} F_{i}=\emptyset$. The last contradiction implies that $S\left(X, R^{*}\right)=\emptyset$. We now pass to the case where $D \neq\{x\}$. It follows that $\{x\} \subset D$. But then, we have for at least one $y_{0} \in X$ that $y_{0} R^{*} x$. Therefore, $y_{0} \in D$, otherwise: since $x \in D$, we cannot have $y_{0} R^{*} x$.

Put

$$
A_{x}=\left\{y \in D \mid(x, y) \in \overline{R^{*}}\right\}
$$

We show that $y_{0} \in A_{x}$ which, together with $\left(y_{0}, x\right) \in R^{*}$, contradicts the acyclicity of $R^{*}$. We first show that $A_{x} \neq \emptyset$. Suppose to the contrary that $A_{x}=\emptyset$. Then, for each $y \in D,(x, y) \notin \overline{R^{*}} \supseteq R^{*}$. It follows that $D \backslash\{x\} \subset D$ is an $R^{*}$-undominated subset of $X$, a contradiction because of the minimal character of $D$. Let $D(x)=D \backslash A_{x}$. We now show that $D(x)=\emptyset$. We proceed by contradiction, so let us assume that $D(x) \neq \emptyset$. Then, for each $t \in A_{x}$ and each $s \in D(x)$ we have $(t, s) \notin R^{*}$ for suppose otherwise, $(t, s) \in R^{*}$ implies that $(x, s) \in \overline{R^{*}}$ contradicting $s \in D(x)$. Therefore, $D(x) \subset D$ is an $R^{*}$-undominated subset of $X$, which is again a contradiction. Hence, $D(x)=\emptyset$ which implies that $A_{x}=D$. Since $y_{0} \in D$, we conclude that $\left(x, y_{0}\right) \in \overline{R^{*}}$, a contradiction with the assumption that $R^{*}$ is acyclic. This contradiction implies that our assumption that $S\left(X, R^{*}\right) \neq \emptyset$ was false. Thus, the sufficiency part of the theorem is proved.

For the necessity part, assume that $X$ is compact. Let $\mathcal{G S C}(X)$ denotes the family of all generalized upper tc-semicontinuous preferences in $X$. Since $X \times X$ belongs to $\mathcal{G S C}(X)$, this family is non-empty. Take an arbitrary generalized upper tc-semicontinuous preference $R$ in $X$. We prove that $S(X, R) \neq \emptyset$. By virtue of Theorem 19 in Andrikopoulos (2012), $S(X, R)$ is equivalent to the set of maximal elements of the transitive closure of $R$. Thus, we must prove that there exists $x_{0} \in X$ such that for each $y \in X$, we have $\left(y, x_{0}\right) \notin P(\bar{R})$. Indeed, suppose to the contrary that for every $x \in X$, there exists $y \in X$ such that $y P(\bar{R}) x$. Since the space is generalized upper tc-semicontinuous, for each $y \in X$, the set $\{x \in X \mid y P(\bar{R}) x\}$ is an open set in $X$. It follows that,

$$
X=\bigcup_{y \in X}\{x \in X \mid y P(\bar{R}) x\} .
$$

Since the space is compact, there exist $y_{1}, \ldots, y_{n}$ such that

$$
X=\bigcup_{i \in\{1, \ldots, n\}}\left\{x \in X \mid y_{i} P(\bar{R}) x\right\} .
$$

Consider the finite set $\left\{y_{1}, \ldots, y_{n}\right\}$. Since $y_{1} \in X$, then there exists $i \in\{1, \ldots, n\}$ such that $y_{i} P(\bar{R}) y_{1}$. If $i=1$, then we have a contradiction. Otherwise, call this element $y_{2}$. We have $y_{2} \overline{P(R)} y_{1}$. Similarly, $y_{3} P(\bar{R}) y_{2} P(\bar{R}) y_{1}$. As $\left\{y_{1}, \ldots, y_{n}\right\}$ is finite, by an induction argument based on this logic, we obtain the existence of a cycle for $P(\bar{R})$. 
This last conclusion contradicts the acyclicity of $P(\bar{R})$. Hence, there exists $x_{0} \in X$ such that for each $y \in X,\left(y, x_{0}\right) \notin P(\bar{R})$. If $x_{0}$ is $R$-undominated, then $x_{0} \in S(X, R)$. Otherwise, there exists $y \in X$ such that $\left(y, x_{0}\right) \in R \subseteq \bar{R}$. Since $\left(y, x_{0}\right) \notin P(\bar{R})$, we conclude that $\left(x_{0}, y\right) \in \bar{R}$. Therefore, $x_{0}$ belongs to an $R$-cycle. By the Lemma of Zorn, the family of all $R$-cycles which contain $x_{0}$ has a maximal element, let $\mathcal{K}\left(x_{0}\right)$. We prove that $\mathcal{K}\left(x_{0}\right)$ is a minimal $R$-undominated set in $X$. Suppose to the contrary, that $(t, s) \in R$ for some $t \in X \backslash \mathcal{K}\left(x_{0}\right)$ and $s \in \mathcal{K}\left(x_{0}\right)$. It follows that $\left(t, x_{0}\right) \in \bar{R}$. Since $\left(t, x_{0}\right) \notin P(\bar{R})$, we conclude that $\left(x_{0}, t\right) \in \bar{R}$. Hence, $\mathcal{K}\left(x_{0}\right) \cup\{t\}$ is an $R$-cycle, a contradiction because of the maximal character of $\mathcal{K}\left(x_{0}\right)$. Therefore, $\mathcal{K}\left(x_{0}\right)$ is an $R$-undominated subset of $X$. It is also minimal for this property as one may verify directly. The last conclusion shows that $S(X, R) \neq \emptyset$.

\section{References}

Alcantud, J.C.R.: Characterization of the existence of maximal elements of acyclic relations. Econ. Theory 19, 407-416 (2002)

Araujo, A., Martins-da-Rocha, V.F., Monteiro, P.K.: Equilibria in reflexive Banach lattices with a continuum of agents. Econ. Theory 24, 469-492 (2004)

Andrikopoulos, A.: On the construction of non-empty choice sets. Soc. Choice Welf. 38, 305-323 (2012)

Andrikopoulos, A.: A representation of consistent binary relations. Span. Econ. Rev. 9, 299-307 (2007)

Brown, D.J.: Acyclic choice. Cowles Foundation Discussion Paper No. 360, Yale University, Mimeo (1973)

de Castro, L.I., Pesce, M., Yannelis, N.C.: Core and equilibria under ambiguity. Econ. Theory 48, 519-548 (2011)

Gutiérrez, J.M.: A characterization of compactness through preferences. Math. Soc. Sci. 57, 131-133 (2009)

Kalai, E., Schmeidler, D.: An admissible set occurring in various bargaining situations. J. Econ. Theory 14(2), 402-411 (1977)

Kelley, J.L.: General Topology. Springer, Berlin (1955)

Prokopovych, P.: On equilibrium existence in payoff secure games. Econ. Theory 48, 5-16 (2011). doi:10.1007/s00199-010-0526-1

Sánchez, M.C., Llinares, J.V., Subiza, B.: A KKM-result and an application for binary and non-binary choice functions. Econ. Theory 21, 185-193 (2003)

Schwartz, T.: The logic of Collective Choice. Columbia University Press, New York (1986)

Sloss, J.: Stable points of directional preference relations. Operations Research House Technical Report, No. 71-7, Stanford University (1971)

Yannelis, N.C., Prabhakar, N.D.: Existence of maximal elements and equilibria in linear topological spaces. J. Econ. Theory 12, 233-245 (1983) 